\title{
RANK-ONE PERTURBATIONS OF DIAGONAL OPERATORS
}

\author{
Eugen J. Ionascu
}

We study rank-one perturbations of diagonal Hilbert space operators mainly from the standpoint of invariant subspace problem. In addition to proving some general properties of these operators, we identify the normal operators and contractions in this class. We show that two well known results about the eigenvalues of rank-one perturbations and one-codimension compressions of self-adjoint compact operators are equivalent. Sufficient conditions are given for existence of nontrivial invariant subspaces for this class of operators.

\section{Preliminaries}

We let $\mathcal{H}$ be a separable, infinite dimensional, complex Hilbert space, and let $\mathcal{L}(\mathcal{H})$ denote the algebra of all bounded linear operators on $\mathcal{H}$. If $u, v \in \mathcal{H}$, we shall write $u \otimes v$ for the operator of rank one defined by

$$
(u \otimes v) x=<x, v>u, \quad x \in \mathcal{H},
$$

where $<,>$ denotes the inner product of the Hilbert space $\mathcal{H}$. The class $\mathcal{N}$ of operators $T$ in $\mathcal{L}(\mathcal{H})$ which can be written in the form $T=N+(u \otimes v)$, where $N$ is a normal operator and $(u \otimes v) \neq 0$ is still not very well understood. Indeed, even the smaller class of operators of the above form, where $N$ is a diagonalizable normal operator, is not in a much better situation, despite the structural simplicity of diagonalizable operators. In this paper we are interested in this second class of operators which will be denoted simply by $\mathcal{D}$. Some spectral properties, examples, applications and the equivalence between two known results about the eigenvalues of rank-one perturbations and one-codimensional compressions of selfadjoint compact operators are discussed in Section 2. We characterize those operators in $\mathcal{D}$ which are normal and prove that under mild assumptions they have the single value extension property in Section 3. In Section 4 we give a characterization for an operator in a relatively natural subclass of $\mathcal{D}$ to be a contraction. Finally in the last section, we combine our previous results with known reductions that one would naturally make in dealing with 
the invariant subspace problem for this class of operators and give a sufficient condition for the existence of a non-trivial invariant subspace based on the Lomonosov's theorem [21].

Similar problems concerning operators in the class $\mathcal{N}$, or rank-one perturbations of different classes of operators such as isometries, selfadjoint compact operators, selfadjoint Toeplitz operators, shift restriction operators, cyclic operators, differential operators, (or Volterra operator) have been studied in a series of papers of which we cite only a few of them: [3], [4], [16]-[19], [22]-[25], [28]-[32]. It is worth mentioning that the class of rankone perturbations of bounded (or unbounded) selfadjoint operators has been extensively studied and many interesting spectral properties have been established in various works (see for instance [8]-[11], [14], [15], [29], [30]).

We let $\left\{e_{n}\right\}_{n=1}^{\infty}$ denote an orthonormal basis for $\mathcal{H}$ which will remain fixed throughout the paper. We also let $\left\{\lambda_{n}\right\}_{n=1}^{\infty}$ be an arbitrary bounded sequence of complex numbers and throughout the remainder of the paper we shall write $\operatorname{Diag}\left(\left\{\lambda_{n}\right\}\right)$ for the unique operator $D$ satisfying $D e_{n}=\lambda_{n} e_{n}, n \in \mathbb{N}$. We shall denote henceforth by $\mathcal{D}_{0}$ the subset of $\mathcal{L}(\mathcal{H})$ consisting of all operators $T$ which can be written in the form

$$
T=\operatorname{Diag}\left(\left\{\lambda_{n}\right\}\right)+u \otimes v, \quad u \neq 0, v \neq 0 .
$$

Throughout the paper we shall suppose that $u$ and $v$ are nonzero vectors in $\mathcal{H}$ and their expansions with respect to the (ordered, orthonormal) basis $\left\{e_{n}\right\}$ are

$$
u=\sum_{n=1}^{\infty} \alpha_{n} e_{n}, \quad v=\sum_{n=1}^{\infty} \beta_{n} e_{n} .
$$

Note that up to unitary equivalence, $\mathcal{D}_{0}$ consists exactly of all sums $N+R$, where $N$ is a normal operator whose eigenvectors span $\mathcal{H}$ and $R$ is an operator of rank one. Note also that the inclusion $\mathcal{D} \subset \mathcal{N}$ is a strict one. One way to see this is to make use of Kato and Rosenblum's result (cf. [20]) stating that the absolutely continuous parts of a selfadjoint operator and its selfadjoint trace class perturbation are unitarily equivalent.

Observe that the expression for $T$ in (1) is not necessarily unique. If we restrict our study, though, to the class $\mathcal{D}_{1}$ of those operators in $\mathcal{D}_{0}$ which admit a representation as in (1) with $u$ and $v$ having nonzero components $\alpha_{n}$ and $\beta_{n}$ for all $n \in \mathbb{N}$, we have uniqueness in the following sense.

Proposition 1.1. If $T \in \mathcal{D}_{1}$ then the representation (1) for $T$ is unique in the sense that if $T=\operatorname{Diag}\left(\left\{\lambda_{n}\right\}\right)+(u \otimes v)=\operatorname{Diag}\left(\left\{\lambda_{n}^{\prime}\right\}\right)+\left(u^{\prime} \otimes v^{\prime}\right)$, then $\operatorname{Diag}\left(\left\{\lambda_{n}\right\}\right)=$ $\operatorname{Diag}\left(\left\{\lambda_{n}^{\prime}\right\}\right)$ and $(u \otimes v)=\left(u^{\prime} \otimes v^{\prime}\right)$.

Proof. We may assume $T=\operatorname{Diag}\left(\left\{\lambda_{n}\right\}\right)+(u \otimes v)=\operatorname{Diag}\left(\left\{\lambda_{n}^{\prime}\right\}\right)+\left(u^{\prime} \otimes v^{\prime}\right)$ where all the Fourier coefficients of $u$ and $v$ in (2) are not zero. This means that $\operatorname{Diag}\left(\left\{\lambda_{n}\right\}\right)-$ $\operatorname{Diag}\left(\left\{\lambda_{n}^{\prime}\right\}\right)=\operatorname{Diag}\left(\left\{\lambda_{n}-\lambda_{n}^{\prime}\right\}\right)=\left(u^{\prime} \otimes v^{\prime}\right)-(u \otimes v)$ has rank at most two. Thus, there exist different positive integers $n_{1}, n_{2}$ such that $\lambda_{k}=\lambda_{k}^{\prime}$ for all $k \in \mathbb{N} \backslash\left\{n_{1}, n_{2}\right\}$. Moreover the range of $S=\operatorname{Diag}\left(\left\{\lambda_{n}-\lambda_{n}^{\prime}\right\}\right)$ is contained in $\vee\left\{e_{n_{1}}, e_{n_{2}}\right\}$, and so we may have three 
essentially different situations. If the range of $S$ is (0) we are done. If the range of $S$ is onedimensional - say, spanned by $e_{n_{1}}$, then since $\left(u^{\prime} \otimes v^{\prime}\right)-(u \otimes v)$ would have a two-dimensional range if $\left\{u, u^{\prime}\right\}$ and $\left\{v, v^{\prime}\right\}$ are linearly independent sets of vectors, we get that either $u$ and $u^{\prime}$ are linearly dependent or $v$ and $v^{\prime}$ are. Let us suppose that $u$ and $u^{\prime}$ are linearly dependent. Then $u=\alpha_{n_{1}} e_{n_{1}}$ and $u^{\prime}=\beta_{n_{1}} e_{n_{1}}$. But this cannot happen since we have assumed that $\left\langle u, e_{k}>\neq 0\right.$ for all $k \in \mathbb{N}$. Similarly the case in which $v$ and $v^{\prime}$ are linearly dependent is ruled out. If the range of $S$ were two-dimensional, then $\vee\left\{u, u^{\prime}\right\}=\vee\left\{e_{n_{1}}, e_{n_{2}}\right\}$, and again we would have a contradiction.

\section{SpeCtral PROperties}

The next two propositions show that when looking for nontrivial invariant subspaces for operators in $\mathcal{D}_{0}$, one can then restrict his attention to the subset $\mathcal{D}_{2}$ of $\mathcal{D}_{1}$ consisting of those operators $T=D+(u \otimes v)$ in $\mathcal{D}_{1}$ such that $D$ has uniform multiplicity one (i.e., if $D=\operatorname{Diag}\left(\left\{\lambda_{n}\right\}\right)$, then all of the numbers $\lambda_{n}, n \in \mathbb{N}$, are pairwise distinct).

Proposition 2.1. Suppose $T=\operatorname{Diag}\left(\left\{\lambda_{n}\right\}\right)+(u \otimes v) \in \mathcal{D}_{0}$ is not a normal operator, and for some $n_{0} \in \mathbb{N}, \alpha_{n_{0}}=0$ or $\beta_{n_{0}}=0$. Then $T^{*}[$ resp. T] has point spectrum and $T$ and $T^{*}$ have nontrivial hyperinvariant subspaces (n.h.s).

Proof. In case $\alpha_{n_{0}}=<u, e_{n_{0}}>=0$, we have

$$
T^{*} e_{n_{0}}=\bar{\lambda}_{n_{0}} e_{n_{0}}+(v \otimes u) e_{n_{0}}=\bar{\lambda}_{n_{0}} e_{n_{0}}+<e_{n_{0}}, u>v=\bar{\lambda}_{n_{0}} e_{n_{0}},
$$

which shows that $\sigma_{p}\left(T^{*}\right)$, the point spectrum of $T^{*}$, is nonempty, and since $T^{*}$ is non-normal, the eigenspace associated with $\bar{\lambda}_{n_{0}}$ is a n.h.s. for $T^{*}$. Its orthogonal complement is thus hyperinvariant for $T$. The case $\beta_{n_{0}}=0$ is handled similarly.

For a diagonal operator $D=\operatorname{Diag}\left(\left\{\lambda_{n}\right\}\right)$ we denote by $\Lambda(D)$ the set of all its eigenvalues $\lambda_{n}$.

Proposition 2.2. If $T=D+(u \otimes v) \in \mathcal{D}_{1}$ and at least one $\lambda \in \Lambda(D)$ has multiplicity larger than 1 , then $T$ has $\lambda$ in its point spectrum.

Proof. Suppose $\lambda=\lambda_{n_{0}}=\lambda_{n_{1}}, n_{0} \neq n_{1}$. Then $(T-\lambda) e_{\lambda_{n_{0}}}=\left\langle e_{\lambda_{n_{0}}}, v\right\rangle$ $u=\overline{\beta_{n_{0}}} u$, and $(T-\lambda) e_{\lambda_{n_{1}}}=<e_{\lambda_{n_{1}}}, v>u=\overline{\beta_{n_{1}}} u$. Hence, if $\beta_{n_{0}} \neq 0$ and $\beta_{n_{1}} \neq 0$ then $(T-\mu)\left(\overline{\beta_{n_{1}}} e_{\lambda_{n_{0}}}-\overline{\beta_{n_{0}}} e_{\lambda_{n_{1}}}\right)=0$. In any case $T-\lambda$ is not injective, and then $\lambda \in \sigma_{p}(T)$.

For an operator $T \in \mathcal{D}_{1}$ given by (1), an interesting phenomenon happens with the isolated eigenvalues of $\operatorname{Diag}\left(\lambda_{n}\right)$ : they are not in the spectrum of $T$. The following theorem gives necessary and sufficient conditions for a point $\mu$ in $\sigma(D)\left(T=D+(u \otimes v) \in \mathcal{D}_{0}\right)$ to be in $\varrho(T)$ (resolvent set).

TheOREm 2.3. Suppose we have $T=D+(u \otimes v) \in \mathcal{D}_{0}$ and $\mu \in \sigma(D)$. Then $\mu \in \varrho(T)$ if and only if the following two conditions are satisfied:

(i) $\mu$ is an isolated eigenvalue of $D, \lambda_{n_{0}}$, of multiplicity one,

(ii) $\beta_{n_{0}}=<v, e_{n_{0}}>\neq 0$ and $\alpha_{n_{0}}=<u, e_{n_{0}}>\neq 0$. 
Proof. For the necessity part of this theorem, let us assume first that (i) is not satisfied. We have three cases: (a) $\mu$ is not an eigenvalue; (b) $\mu$ is an eigenvalue but is not isolated, and (c) $\mu$ is an isolated eigenvalue but has multiplicity larger than 1 . In the cases (a) and (b), there exists a sequence of distinct eigenvalues $\left\{\lambda_{n_{k}}\right\}_{k \geq 1}$ such that $\lambda_{n_{k}} \rightarrow \mu$. Then, since $(T-\mu) e_{\lambda_{n_{k}}}=\left(\lambda_{n_{k}}-\mu\right) e_{\lambda_{n_{k}}}+<e_{\lambda_{n_{k}}}, v>u$ we have

$$
\left\|(T-\mu) e_{\lambda_{n_{k}}}\right\| \leq\left|\lambda_{n_{k}}-\mu\right|+\left|<e_{\lambda_{n_{k}}}, v>\right|\|u\| \rightarrow 0,
$$

as $k$ goes to infinity. This says in particular that $T-\mu$ is not bounded below (if it is injective), and then it cannot be invertible. In other words, $\mu \in \sigma(T)$. In the case (c), if we have $\mu=\lambda_{n_{0}}=\lambda_{n_{1}}$, then $(T-\mu) e_{\lambda_{n_{0}}}=\left\langle e_{\lambda_{n_{0}}}, v>u=\overline{\beta_{n_{0}}} u\right.$, and $(T-\mu) e_{\lambda_{n_{1}}}=\left\langle e_{\lambda_{n_{1}}}, v>\right.$ $u=\overline{\beta_{n_{1}}} u$. Hence, if $\beta_{n_{0}} \neq 0$ and $\beta_{n_{1}} \neq 0$ then $(T-\mu)\left(\overline{\beta_{n_{1}}} e_{\lambda_{n_{0}}}-\overline{\beta_{n_{0}}} e_{\lambda_{n_{1}}}\right)=0$. In any case $T-\mu$ is not injective, and then again $\mu \in \sigma(T)$.

Suppose now that (i) holds but (ii) doesn't. First, if $\beta_{n_{0}}=0$, we get as above $(T-\mu) e_{\lambda_{n_{0}}}=0$, and so $\mu \in \sigma(T)$. If $\alpha_{n_{0}}=0$, then $\left(T^{*}-\bar{\mu}\right) e_{\lambda_{n_{0}}}=0$, and then $\bar{\mu} \in \sigma\left(T^{*}\right)$, or equivalently, $\mu \in \sigma(T)$.

For the sufficiency, we assume now that (i) and (ii) hold. We want to show that $T-\mu$ is invertible. Since $\mu$ is an isolated point in $\sigma(D)$ and $D$ is normal, $D-\mu$ and hence $T-\mu$, is Fredholm with index zero. Thus it suffices to show that $\mu$ is not an eigenvalue for $T$. If $(T-\mu) x=\left(D-\lambda_{n_{0}}\right) x+\left\langle x, v>u=0\right.$, then by our hypothesis, $\alpha_{n_{0}} \neq 0$, it follows that $\langle x, v\rangle=0$. So, $x=\gamma e_{n_{0}}$ with $\gamma \neq 0$, and this contradicts the hypothesis $\beta_{n_{0}} \neq 0$.

We characterize now the point spectrum of an operator $T$ in $\mathcal{D}_{1}\left[\operatorname{resp} . \mathcal{D}_{2}\right]$.

Proposition 2.4. For $\lambda \in \mathbb{C}, \lambda$ is an eigenvalue for $T=D+(u \otimes v) \in \mathcal{D}_{1}$ if and only if

(i) $u \in \operatorname{Range}(D-\lambda)$, and

(ii) $\langle x, v\rangle+1=0$ for at least one vector $x \in \mathcal{H}$ satisfying $u=(D-\lambda) x$.

Equivalently, $\lambda$ is an eigenvalue for $T=\operatorname{Diag}\left(\left\{\lambda_{n}\right\}\right)+(u \otimes v) \in \mathcal{D}_{2}$ if and only if

(iii) $\lambda \notin \Lambda(D)$,

(iv) $\sum_{\lambda_{n} \in \Lambda(D)} \frac{\left|\alpha_{n}\right|^{2}}{\left|\lambda-\lambda_{n}\right|^{2}}<\infty$, and

(v) $\sum_{\lambda_{n} \in \Lambda(D)} \frac{\alpha_{n} \overline{\beta_{n}}}{\lambda-\lambda_{n}}=1$

Proof. For the necessity part, let $\lambda \in \mathbb{C}$ be an eigenvalue for $T$ and $x \in \mathcal{H} \backslash\{0\}$, such that $T x=\lambda x$. Then $\langle x, v\rangle u=(\lambda-D) x$. We cannot have $\langle x, v\rangle=0$ because we obtain then $\lambda=\lambda_{i_{0}}, x=\xi e_{i_{0}}, \xi \in \mathbb{C} \backslash\{0\}$, and then $\overline{\beta_{i_{0}}}=<e_{i_{0}}, v>=\frac{1}{\xi}<x, v>=0$ which is not possible since $T \in \mathcal{D}_{1}$. Hence, if we write $\widetilde{x}=-\frac{1}{<x, v>} x$, then $u=(D-\lambda) \widetilde{x}$ and $\langle\widetilde{x}, v>+1=0$.

For the sufficiency part, we can assume that there exists $x \in \mathcal{H}$ such that $u=$ $(D-\lambda) x$ and $\langle x, v\rangle+1=0$. Then $x \neq 0$ and $T x=D x+<x, v>u=u+\lambda x-u=\lambda x$. 
Finally, suppose (i) is valid and $\lambda \in \Lambda(D)$. Then $u=(D-\lambda I) x=\left(D-\lambda_{n_{0}}\right) x$ for some $x \in \mathcal{H}$, and so $\alpha_{n_{0}}=0$ which contradicts that $T \in \mathcal{D}_{1}$. It follows that $\lambda \notin \Lambda(D)$ and the rest of the equivalence between (i) together with (ii) and (iii)-(v) is now obvious.

For $T=D+u \otimes v \in \mathcal{D}_{1}$, the diagonal operator $D$ and the rank-one operator are uniquely determined by $T$ (Theorem 1.1) and so we can define the function $f_{T}(z)=<$ $(z I-D)^{-1} u, v>$, for $z \in \mathbb{C} \backslash \overline{\Lambda(D)}$. This function is clearly an analytic function and it can be written as a Borel series ([2]):

$$
f_{T}(z)=\sum_{n=1}^{\infty} \frac{\alpha_{n} \overline{\beta_{n}}}{z-\lambda_{n}}, \quad z \in \mathbb{C} \backslash \overline{\Lambda(D)} .
$$

Corollary 2.5. Assume $T=D+u \otimes v \in \mathcal{D}_{1}$ and $\lambda \in \mathbb{C} \backslash \overline{\Lambda(D)}$. Then $\lambda$ is an eigenvalue for $T$ if and only if $f_{T}(\lambda)=1$.

Proof. Since $\lambda \in \mathbb{C} \backslash \overline{\Lambda(D)}$, part (i) in Proposition 2.4 is satisfied. Taking $x=(D-\lambda I)^{-1} u$ in part (ii) of Proposition 2.4 we obtain the corollary.

The next corollary describes the spectrum of an operator $T \in \mathcal{D}_{2}$.

Corollary 2.6. If $T=D+(u \otimes v) \in \mathcal{D}_{2}$ then

$$
\sigma(T)=\Lambda(D)^{\prime} \cup\left\{z \in \mathbb{C} \backslash \overline{\Lambda(D)} ; f_{T}(z)=1\right\},
$$

where $\Lambda(D)^{\prime}$ denotes the derived set of $\Lambda(D)$.

Proof. In general for an operator $A \in \mathcal{L}(\mathcal{H}), \sigma(A)=\sigma_{e}(A) \cup \sigma_{p}(A) \cup \sigma_{p}\left(A^{*}\right)^{*}$, where if $\Delta \subset \mathbb{C}, \Delta^{*}=\{\bar{z}: z \in \Delta\}$ (cf. [6], p. 51). Since $T \in \mathcal{D}_{2}$, we have $\sigma_{e}(T)=\sigma_{e}(D)=$ $\Lambda(D)^{\prime}$, and so by Corollary 2.5, one inclusion necessary to establish (4) follows. For the other inclusion, let us assume $\lambda \in \sigma(T)=\sigma_{e}(T) \cup \sigma_{p}(T) \cup \sigma_{p}\left(T^{*}\right)^{*}$. Since $\sigma_{e}(T)=\Lambda(D)^{\prime}$, we can assume that $\lambda \notin \sigma_{e}(T)$. Suppose then that $\lambda \in \sigma_{p}(T)$. If $\lambda \in \sigma_{p}(T) \cap \overline{\Lambda(D)}$, by Proposition 2.4, $\lambda \notin \Lambda(D)$ and so $\lambda \in \Lambda(D)^{\prime}=\sigma_{e}(T)$ which contradicts our assumption. It follows that $\lambda \in \sigma_{p}(T) \backslash \overline{\Lambda(D)}$ and so by Corollary 2.5, $f_{T}(\lambda)=1$. Since $f_{T}(z)=\overline{f_{T^{*}}(\bar{z})}$, for all $z \in \mathbb{C} \backslash \overline{\Lambda(D)}$, one takes care likewise of the case $\lambda \in \sigma_{p}\left(T^{*}\right)^{*}$.

Example ([33]) Let $T=\operatorname{Diag}\left(\lambda_{n}\right)+u \otimes u$ where $D=\operatorname{Diag}\left(\lambda_{n}\right)$ and $u$ are constructed in the following way. First we consider a family of open disjoint (and non tangent) disks $\left\{D_{n}\right\}_{n \in \mathbb{N}}\left(D_{n}\right.$ is centered at $\lambda_{n}$ and has radius $\left.r_{n}\right)$ contained in the unit disk $\mathbb{D}=\{z \in \mathbb{C}:|z|<1\}$ and such that the set $\mathbb{D} \backslash \cup_{n \in \mathbb{N}} \overline{D_{n}}$ has Lebesgue measure zero. Such a family can be constructed using an induction argument, covering at each step a closed set of whose measure is a fixed nonzero fraction of the measure of the open set uncovered by the disks constructed at previous steps.

The diagonal operator $D$ is defined by the sequence $\left\{\lambda_{n}\right\}$ constructed above and $u, v$ are given as in (2) where $\alpha_{n}=\beta_{n}=r_{n}, n \in \mathbb{N}$. We want to compute the point spectrum of $T$. In order to do this let us observe that the essential spectrum of $T$ is 
$\Lambda(D)^{\prime}=\overline{\mathbb{D}} \backslash \cup_{n \in \mathbb{N}} D_{n}$. Also we need the following formula which can be proved easily by a change of variables to polar coordinates:

$$
\iint_{D(a, r)} \frac{d x d y}{z-(x+y \mathrm{i})}= \begin{cases}\frac{\pi r^{2}}{z-a}, & \text { if } \quad|z-a|<r \\ \pi(\bar{z}-\bar{a}), & \text { if } \quad|z-a| \leq r\end{cases}
$$

for every $a \in \mathbb{C}$ and $r>0$. Then if $z \notin \overline{\mathbb{D}}$, by (3) and (5), we have

$$
f_{T}(z)=\sum_{k \in \mathbb{N}} \frac{r_{k}^{2}}{z-\lambda_{k}}=\frac{1}{\pi} \sum_{k \in \mathbb{N}} \iint_{D\left(\lambda_{k}, r_{k}\right)} \frac{d x d y}{z-(x+y \mathrm{i})}=\frac{1}{\pi} \iint_{\mathbb{D}} \frac{d x d y}{z-(x+y \mathrm{i})}=\frac{1}{z} .
$$

Hence, by Proposition 2.4, $T$ does not have any eigenvalues $z \in \mathbb{C} \backslash \overline{\mathbb{D}}$. Let us suppose that $z \in \overline{\mathbb{D}} \backslash \cup_{n \in \mathbb{N}} \bar{D}_{n}$. In this case if $z$ were an eigenvalue for $T$ then by Proposition 2.4 , the sum $\sum_{k \in \mathbb{N}} \frac{r_{k}^{2}}{\left|z-\lambda_{k}\right|^{2}}$ would be convergent and $\sum_{k \in \mathbb{N}} \frac{r_{k}^{2}}{z-\lambda_{k}}=1$. But using again (5), we have

$$
\sum_{k \in \mathbb{N}} \frac{r_{k}^{2}}{z-\lambda_{k}}=\frac{1}{\pi} \sum_{k \in \mathbb{N}_{D}} \iint_{D\left(\lambda_{k}, r_{k}\right)} \frac{d x d y}{z-(x+y \mathrm{i})}=\frac{1}{\pi} \iint_{\mathbb{D}} \frac{d x d y}{z-(x+y \mathrm{i})}=\bar{z} .
$$

This implies that the only possible point which may be an eigenvalue in this cases is $z=1$. Hence, $z=1$ is an eigenvalue if and only if $\sum_{k \in \mathbb{N}} \frac{r_{k}^{2}}{\left|1-\lambda_{k}\right|^{2}}<\infty$. Suppose $z \in \bar{D}_{n} \backslash\left\{\lambda_{n}\right\}$ for some $n \in \mathbb{N}$ and let us assume that $z$ is an eigenvalue for $T$. Then using (5) again we can compute

$$
\sum_{k \in \mathbb{N}} \frac{r_{k}^{2}}{z-\lambda_{k}}=\frac{1}{\pi} \sum_{k \in \mathbb{N}, k \neq \lambda_{n}} \iint_{D\left(\lambda_{k}, r_{k}\right)} \frac{d x d y}{z-(x+y \mathrm{i})}+\frac{r_{n}^{2}}{z-\lambda_{n}}=\bar{z}-\left(\bar{z}-\overline{\lambda_{n}}\right)+\frac{r_{n}^{2}}{z-\lambda_{n}}=\overline{\lambda_{n}}+\frac{r_{n}^{2}}{z-\lambda_{n}} .
$$

This shows that $z=\lambda_{n}+\frac{r_{n}^{2}}{1-\overline{\lambda_{n}}}$ is the only possible eigenvalue for $T$ in this case. In fact, it is easy to see that these values are indeed eigenvalues for $T$. Hence,

$$
\sigma_{p}(T)=\left\{\lambda_{n}+\frac{r_{n}^{2}}{1-\overline{\lambda_{n}}}: n \in \mathbb{N}\right\} \cup A
$$

where $A=\{1\}$ if $\sum_{k \in \mathbb{N}} \frac{r_{k}^{2}}{\left|1-\lambda_{k}\right|^{2}}<\infty$ and $A=\emptyset$ if $\sum_{k \in \mathbb{N}} \frac{r_{k}^{2}}{\left|1-\lambda_{k}\right|^{2}}=\infty$.

A natural question which arises at this point is whether or not there exist operators $T \in \mathcal{D}_{0}$ with empty point spectrum. An example of such an operator was first constructed by J. G. Stampfli in [31], for the case when the spectrum of $T$ is a square. Given an arbitrary nonempty compact subset of the plane $K$, it is interesting to know if there are examples of operators $T \in \mathcal{D}_{0}$ with empty point spectrum and such that $\sigma(T)=K$. Next, we put together some information about the resolvent of operators $T$ in $\mathcal{D}_{2}$ around points which are isolated in $\Lambda(D)$.

Lemma 2.7. Let $A \in \mathcal{L}(\mathcal{H})$ be an invertible operator, and let $S=A+(u \otimes v)$. Then $S$ is invertible if and only if $\left\langle A^{-1} u, v\right\rangle+1 \neq 0$, and its inverse is given by the 
formula

$$
S^{-1}=A^{-1}-\frac{1}{<A^{-1} u, v>+1}\left(A^{-1} u \otimes\left(A^{*}\right)^{-1} v\right) .
$$

In particular, if $T=D+(u \otimes v) \in \mathcal{D}_{1}$ and $f_{T}(\lambda) \neq 1$ for some $\lambda \in \mathbb{C} \backslash \overline{\Lambda(D)}$, we have

$$
(\lambda-T)^{-1}=
$$

$$
(\lambda-D)^{-1}-\left(f_{T}(\lambda)-1\right)^{-1}\left((\lambda-D)^{-1} u \otimes\left((\lambda-D)^{*}\right)^{-1} v\right) .
$$

Proof. If $\left\langle A^{-1} u, v\right\rangle+1=0$, then $u \neq 0$ and since $S\left(A^{-1} u\right)=0$, it is clear that $S$ is not invertible. On the other hand, if $\left\langle A^{-1} u, v\right\rangle+1 \neq 0$, then it is enough to check that (6) gives the inverse of $S$ :

$$
\begin{array}{r}
{[A+(u \otimes v)]\left[A^{-1}-\frac{1}{<A^{-1} u, v>+1}\left(A^{-1} u \otimes\left(A^{*}\right)^{-1} v\right)\right]=I+\left(u \otimes\left(A^{*}\right)^{-1} v\right)-} \\
\frac{1}{<A^{-1} u, v>+1}\left(u \otimes\left(A^{*}\right)^{-1} v\right)-\frac{<A^{-1} u, v>}{<A^{-1} u, v>+1}\left(u \otimes\left(A^{*}\right)^{-1} v\right)=I .
\end{array}
$$

The second part of the lemma clearly follows from the first part.

For $T=D+u \otimes v \in \mathcal{D}_{0}$ we define the function $F_{T}(z)=<(z I-T)^{-1} u, v>$ for $z \in \mathbb{C} \backslash \sigma(T)$. We have the following relation between the functions $F_{T}$ and $f_{T}$.

Proposition 2.8. Assume $T=D+(u \otimes v) \in \mathcal{D}_{1}$. Then for all $z \in \mathbb{C} \backslash(\sigma(T) \cup \sigma(D))$ we have

$$
F_{T}(z)=\frac{f_{T}(z)}{1-f_{T}(z)}
$$

Moreover, if $\zeta \in \Lambda(D) \backslash \Lambda(D)^{\prime}\left(\zeta=\lambda_{n_{0}}\right)$, then $F_{T}(\zeta)=-1, \frac{d F_{T}}{d z}(\zeta)=-\left(\alpha_{n_{0}} \bar{\beta}_{n_{0}}\right)^{-1}$, and if $T \in \mathcal{D}_{2}$ we have

$$
(T-\zeta)^{-1}=\widetilde{D}-\frac{1}{\alpha_{n_{0}}} \widetilde{D} u \otimes e_{n_{0}}-\frac{1}{\bar{\beta}_{n_{0}}} e_{n_{0}} \otimes \widetilde{D}^{*} v+
$$

$$
\left(\alpha_{n_{0}} \bar{\beta}_{n_{0}}\right)^{-1}\left(\sum_{k \neq n_{0}} \frac{\alpha_{k} \bar{\beta}_{k}}{\lambda_{k}-\zeta}+1\right) e_{n_{0}} \otimes e_{n_{0}},
$$

where $\widetilde{D}=\sum_{k \neq n_{0}}\left(\lambda_{k}-\zeta\right)^{-1} e_{k} \otimes e_{k}$.

Proof. Formula (8) can be easily derived from (7). Each $\zeta \in \Lambda(D) \backslash \Lambda(D)^{\prime}$ is an isolated eigenvalue of multiplicity one for $D$, and hence by Theorem $2.3, T-\zeta$ is invertible. We have $\zeta-D=\zeta-T+(u \otimes v)$ and then by Lemma $2.7,<(\zeta-T)^{-1} u, v>+1=0$, which proves that $F_{T}(\zeta)=-1$. To compute $\frac{d F_{T}}{d z}(\zeta)$ we differentiate $(8)$ at a point $z$ different of $\zeta$ and take the limit as $z \rightarrow \zeta$ :

$$
\frac{d F_{T}}{d z}(\zeta)=\lim _{z \rightarrow \zeta} \frac{f_{T}^{\prime}(z)}{\left(1-f_{T}(z)\right)^{2}}=\lim _{z \rightarrow \zeta} \frac{(z-\zeta)^{2} f_{T}^{\prime}(z)}{\left[z-\zeta-(z-\zeta) f_{T}(z)\right]^{2}}=-\left(\alpha_{n_{0}} \bar{\beta}_{n_{0}}\right)^{-1} .
$$

The equality (9) follows from (7) by a similar argument of passing to the limit as $z \rightarrow \zeta$.

As an application to formula (9) we will show the equivalence of two interesting facts from the theory of selfadjoint compact operators. The first result appears in [32] (see 
also [16]) and the second result was proved independently by several authors (cf. [7], [13] and $[26])$.

TheOREM 2.9. (i) Let $\left\{\nu_{k}\right\}_{k \in \mathbb{N}}$ and $\left\{\mu_{k}\right\}_{k \in \mathbb{N}}$ be two distinct monotone increasing sequences of real numbers, each having zero as the limit point. Further assume that $\left\{\mu_{k}\right\}$ belongs to $\left(\nu_{k}, \nu_{k+1}\right)$ for each $k \in \mathbb{N}$. Then if $A$ is a selfadjoint compact operator on a separable Hilbert space $H$ having the sequence $\nu_{k}(k \in \mathbb{N})$ as its eigenvalues (with multiplicity one), there exists a vector $x \in H$ such that $A+x \otimes x$ has precisely the eigenvalues $\left\{\mu_{k}\right\}_{k \in \mathbb{N}}$.

(ii) Let $\left\{\nu_{k}\right\}_{k \in \mathbb{N}}$ and $\left\{\mu_{k}\right\}_{k \in \mathbb{N}}$ be two distinct monotone decreasing sequences of real numbers, each having zero as the limit point and such that $\left\{\mu_{k}\right\}$ belongs to $\left(\nu_{k+1}, \nu_{k}\right)$ for each $k \in \mathbb{N}$. Then if $A$ is a selfadjoint compact operator on a Hilbert space $H$ having the eigenvalues $\nu_{k}(k \in \mathbb{N}$ ) (with multiplicity one), there exists a vector $y \in H$ such that if $P$ denotes the orthogonal projection on the one-dimensional space spanned by the vector $y$, the compact operator $(I-P) A(I-P)_{\mid(I-P)(H)}$ has exactly as its eigenvalues the sequence $\left\{\mu_{k}\right\}_{k \in \mathbb{N}}$.

Proof. For the implication (i) $\Rightarrow$ (ii) we assume that $\left\{\nu_{k}\right\}_{k \in \mathbb{N}},\left\{\mu_{k}\right\}_{k \in \mathbb{N}}$ and $A$ are as in (ii) and let us take the diagonal operator $D$ on $H$ whose eigenvalues are $\left\{\lambda_{k}\right\}_{k \in \mathbb{N}}$ where $\lambda_{1}=-1, \lambda_{k+1}=\left(1+\mu_{k}\right)^{-1}-1$ for $k \in \mathbb{N}$. Then by $(i)$ we can find $x$ such that $T=D+x \otimes x$ has exactly the eigenvalues $\left\{\left(1+\nu_{k}\right)^{-1}-1\right\}_{k \in \mathbb{N}}$. We take $\zeta=\lambda_{1}$ and apply formula (9) for $D, u=v=x$ and $n_{0}=1$. Let $Q$ be the orthogonal projection on $e_{1}$. We see that $(I-Q)(T-\zeta I)^{-1}(I-Q)_{\mid(I-Q)(H)}$ is a diagonal whose eigenvalues are precisely $\left\{\frac{1}{\mu_{k}-\zeta}\right\}_{k \geq 2}=\left\{1+\mu_{k}\right\}_{k \in \mathbb{N}}$. Hence, by spectral mapping theorem the operator $S=(T-\zeta I)^{-1}-I$ is compact and has the eigenvalues $\left\{\nu_{k}\right\}_{k \in \mathbb{N}}$. Thus, we can find an unitary operator $U$ such that $U^{*} S U=A$. To finish the proof we take $y=U^{*} e_{1}$ and observe that $(I-P) A(I-P)=U^{*}(I-Q) S(I-Q) U$, where $P$ is the orthogonal projection on the one-dimensional space spanned by $y$.

For the implication (ii) $\Rightarrow(\mathrm{i})$, let $\left\{\nu_{k}\right\}_{k \in \mathbb{N}},\left\{\mu_{k}\right\}_{k \in \mathbb{N}}$ and $A$ be as in (i). Without loss of generality, we can assume that $A$ is a diagonal operator with respect to the basis $\left\{e_{k}\right\}_{k \in \mathbb{N}}$ and $\nu_{1}=-1$. Let $B$ be an arbitrary compact operator on $H$ which has $\left\{\left(\mu_{k}+1\right)^{-1}-1\right\}_{k \in \mathbb{N}}$ has its only eigenvalues (multiplicity one). Using (ii) we can find $y:=y_{1} \in H$ such that $(I-P) A(I-P)_{\mid(I-P)(H)}$ has precisely $\left\{\left(\nu_{k+1}+1\right)^{-1}-1\right\}_{k \in \mathbb{N}}$ as its eigenvalues. Let $\left\{y_{k+1}\right\}_{k \in \mathbb{N}}$ be an orthonormal basis in $(I-P)(H)$ with respect to which $(I-P) A(I-P)_{\mid(I-P)(H)}$ diagonalizes. Then the matrix of $B+I$ with respect to the basis $\left\{y_{k}\right\}_{k \in \mathbb{N}}$ looks exactly as the right hand side of (9) (for $D=A, \lambda_{k}=\nu_{k}(k \in \mathbb{N}), \zeta=\nu_{1}, u=v$ and $e_{n_{0}}=1$ ). We shall show that we can determine the coefficients of $u$ such that these two matrices coincide (which will give a unitarily equivalence between the operators which admit this same representation matrix in different orthonormal basis). Let us write the representation 
of $B$ as follows

$$
B+I=b_{1} e_{1} \otimes y_{1}+\sum_{k \geq 2} b_{k} y_{1} \otimes y_{k}+\sum_{k \geq 2} \overline{b_{k}} y_{k} \otimes y_{1}+\sum_{k \geq 2}\left(\nu_{k+1}+1\right)^{-1} y_{k} \otimes y_{k} .
$$

If we compare this with (9) we obtain that $\alpha_{k}=-\alpha_{1}\left(\nu_{k}+1\right) b_{k},(k \geq 2)$ and then

$$
\frac{1}{\left|\alpha_{1}\right|^{2}}=b_{1}-\sum_{k \geq 2}\left(\nu_{k}+1\right)\left|b_{k}\right|^{2} \text {. }
$$

This will allow us to solve for $\alpha_{1}$ if the right hand side of (10) is not zero. Suppose by way of contradiction that this is not true. Then a simple computation shows that $(B+I) z=0$ where $z=y_{1}-\sum_{k \geq 2}\left(\nu_{k}+1\right) \overline{b_{k}}$ and so $B+I$ admits the value 0 as one of its eigenvalues but by our assumption the only eigenvalues of $B+I$ are the elements of the sequence $\left\{\left(\mu_{k}+1\right)^{-1}\right\}_{k \in \mathbb{N}}$. This proves that we have a solution for $u \in H$ and so by spectral theorem $A+u \otimes u$ has precisely the eigenvalues $\left\{\mu_{k}\right\}_{k \in \mathbb{N}}$.

\section{Normality, DeComposability, AND The SVEP}

We begin this section with a characterization of normal operators in $\mathcal{N}_{0}$ which, in particular, applies to the normal operators in $\mathcal{D}_{0}$.

Proposition 3.1. Let $T=N+(u \otimes v) \in \mathcal{L}(\mathcal{H})$, where $N$ is a normal operator and $u, v$ are nonzero vectors in $\mathcal{H}$. Then $T$ is a normal operator if and only if either

(i) $u$ and $v$ are linearly dependent and $u$ is an eigenvector for $\Im\left(\alpha N^{*}\right)$, where $\alpha=\frac{\langle u, v>}{\|v\|^{2}}$, or

(ii) $u, v$ are linearly independent vectors and there exist $\alpha, \beta \in \mathbb{C}$ such that

$$
\begin{gathered}
\left(N^{*}-\bar{\alpha} I\right) u=\|u\|^{2} \beta v \quad \text { and } \\
(N-\alpha I) v=\|v\|^{2} \bar{\beta} u,
\end{gathered}
$$

where $\Re(\beta)=-1 / 2$.

Proof. We observe that the equation $T^{*} T=T T^{*}$ is equivalent to

$$
\begin{gathered}
N^{*} u \otimes v+v \otimes N^{*} u+\|u\|^{2} v \otimes v= \\
N v \otimes u+u \otimes N v+\|v\|^{2} u \otimes u .
\end{gathered}
$$

It is a simple computation to check that (12) is satisfied if (i) or (ii) is true.

Let us assume that $T$ is a normal operator. We distinguish two distinct cases.

CASE I. We assume that $u, v$ are linearly dependent. Thus, there exists $\alpha \in \mathbb{C}$ such that $u=\alpha v\left(\alpha=\left\langle u, v>/\|v\|^{2}\right)\right.$. Since $\|v\|^{2} u \otimes u=|\alpha|^{2}\|v\|^{2} v \otimes v=\|u\|^{2} v \otimes v$, if we write $w=\left(\alpha N^{*}-\bar{\alpha} N\right) v\left(=2 \Im\left(\alpha N^{*}\right) v\right),(12)$ becomes $w \otimes v=-v \otimes w$. This last equality holds if and only if $w=i t v$ for some $t \in \mathbb{R}$ and (i) is proved.

CASE II. We assume that $u, v$ are linearly independent vectors. From (12) we get that

$$
<x, N^{*} u>v=<x, N v>u, \quad x \in(\vee\{u, v\})^{\perp} .
$$


Hence $<N^{*} u, x>=<N v, x>=0$ for every $x \in(\vee\{u, v\})^{\perp}$, which means that

$$
N^{*} u=a_{11} u+a_{12} v, N v=a_{21} u+a_{22} v,
$$

for some $a_{i j} \in \mathbb{C}$. Substituting in (12) we obtain that the $a_{i j}$ satisfy the following relations:

$$
a_{11}=\overline{a_{22}}, \quad a_{12}+\overline{a_{12}}+\|u\|^{2}=a_{21}+\overline{a_{21}}+\|v\|^{2}=0 .
$$

So, if we write $a_{11}=\bar{\alpha}$ and $a_{12}=-\|u\|^{2} / 2+i s_{1}, a_{21}=-\|v\|^{2} / 2+i s_{2}$, where $s_{1}, s_{2} \in \mathbb{R}$, (13) implies that $\left(N^{*}-\bar{\alpha} I\right) u=\left(-\|u\|^{2} / 2+i s_{1}\right) v$ and $(N-\alpha I) v=\left(-\|v\|^{2} / 2+i s_{2}\right) u$. Thus $(N-\alpha I)^{*}(N-\alpha I) u=\left(-\|u\|^{2} / 2+i s_{1}\right)\left(-\|u\|^{2} / 2+i s_{2}\right) u$ which implies that $s_{1} /\|u\|^{2}=$ $-s_{2} /\|v\|^{2}$. If we write $t=1 / 4+\left(1 /\|u\|^{4}\right) s_{1}^{2}$ and $\beta=-1 / 2+\operatorname{sign}\left(s_{1}\right) i \sqrt{t-1 / 4}$ then clearly $u$ and $v$ satisfy (11). (Here, we used the notation sign for the real valued function defined by $\operatorname{sign}(x)=1$ if $x>0, \operatorname{sign}(x)=-1$ if $x<0$ and $\operatorname{sign}(0)=0$.)

COROllary 3.2. $T=D+u \otimes v \in \mathcal{D}_{1}$ is normal if and only if either

(a) there exist $\alpha \in \mathbb{C}$ and $t \in \mathbb{R}$ such that $\Lambda(D)$ lies on the line $\{z \in \mathbb{C}: \Im(\alpha \bar{z})=t\}$, and $u=\alpha v$, or

(b) there exist $\alpha \in \mathbb{C}$ and $t \in \mathbb{R}$ such that $\Lambda(D)$ lies on the circle $\{z \in \mathbb{C}:|z-\alpha|=t\}$, $t \in \mathbb{R}$, and

$$
t u /\|u\|=e^{i \vartheta}(D-\alpha I)(v /\|v\|),
$$

where $\vartheta \in[0, \pi)$ is determined by the equation $\Re\left(t e^{i \vartheta} /\|u\|\|v\|\right)=-1 / 2$.

Proof. Suppose that (a) or (b) holds. Then either $\Im\left(\alpha D^{*}\right)=t I$ or $|D-\alpha I|=t I$. If (a) holds then (i) in Proposition 3.1 holds and hence $T$ is a normal operator. If (b) holds then an easy computation shows that (11) holds for $\beta=t e^{i \vartheta} /\|u\|\|v\|$. The two relations in (11) alone imply that (12) holds and so $T$ is normal.

On the other hand if $T$ is normal then, by Proposition 3.1, (i) or (ii) holds. In case (i) is true then $\Im\left(\alpha D^{*}\right) u=t u$ for some $t \in \mathbb{R}$. Thus $\Im\left(\alpha \overline{\lambda_{n}} \alpha_{n}\right)=t \alpha_{n}$ for all $n \in \mathbb{N}$ and since $\alpha_{n} \neq 0$ for every $n$ in $\mathbb{N}$ we obtain that $\Lambda(D)$ is a subset of the line $\{z \in \mathbb{C}: \Im(\alpha \bar{z})=t\}$ and (a) follows. If (ii) holds, we get from (11) that $(D-\alpha I)^{*}(D-\alpha I) v=\|u\|^{2}\|v\|^{2}|\beta|^{2} v$, and by a similar argument as above, we get that $\Lambda(D)$ is a subset of the circle $\{z \in \mathbb{C}:|z-\alpha|=t\}$, where $t=\|u\|\|v\| \| \beta \mid$. Then, the other part of (b) follows easily from (11).

It is worth mentioning that actually if $\Lambda(D)$ is a subset of a line or of a circle then $T=D+u \otimes v$ is a decomposable operator (cf. Theorem 5.2, [5]). Moreover, $T$ has the property (Triang 0 ) (cf. Theorem 6.16, [5]), i.e., for any pair $\mathcal{S}_{1} \subset \mathcal{S}_{2}$ of invariant subspaces for $T$ such that $\operatorname{dim}\left(\mathcal{S}_{1} / \mathcal{S}_{2}\right)>1$ there exists another invariant subspace $\mathcal{S}_{3}$ of $T$ verifying

$$
\mathcal{S}_{1} \underset{\neq}{\subsetneq \mathcal{S}_{2}} \underset{\neq}{\subset} \mathcal{S}_{3} .
$$

Another interesting question about the class $\mathcal{D}_{0}$ is whether we have the decomposability property for operators in $\mathcal{D}_{0}$ whose spectrum is not necessarily an arc of an analytic curve. It is known ([5]) that every decomposable operator has the following property. 
Definition 3.3. We say that an operator $T \in \mathcal{L}(\mathcal{H})$ has the single valued extension property (notation: SVEP) if the only vector-valued analytic function $f: G \rightarrow \mathcal{H}$, where $G$ is an arbitrary open connected subset of $\mathbb{C}$, which satisfies the equality

$$
(T-z I) f(z)=0, \quad z \in G
$$

is the function identically equal to zero.

Proposition 3.4. Every operator $T=D+(u \otimes v) \in \mathcal{D}_{1}$ for which the set $\mathbb{C} \backslash \overline{\Lambda(D)}$ is connected has the SVEP.

Proof. Let $f: G \rightarrow \mathcal{H}$ be an analytic function such that $(T-z I) f(z)=0$ for every $z \in G$. If $G \cap(\mathbb{C} \backslash \overline{\Lambda(D)}) \neq \emptyset$ then by Corollary 2.5, $T-z I$ is invertible for all $z \in(G \backslash \overline{\Lambda(D)}) \backslash\left\{z \in \mathbb{C} \backslash \overline{\Lambda(D)} ; f_{T}(z)=1\right\}$ and so $f(z)=0$ for all $z \in(G \backslash \overline{\Lambda(D)}) \backslash\{z \in$ $\left.\mathbb{C} \backslash \overline{\Lambda(D)} ; f_{T}(z)=1\right\}$. The function $f_{T}$ cannot be identically equal to 1 on the connected set $\mathbb{C} \backslash \overline{\Lambda(D)}$ because $\lim _{|z| \rightarrow \infty} f_{T}(z)=0$. Hence the set $\left\{z ; f_{T}(z)=1\right\}$ is discrete and since $G$ is connected it follows that $f$ is identically zero.

We may assume that actually $G \subset \overline{\Lambda(D)}$. If we expand $f$ in the basis $\left\{e_{n}\right\}$ as $f=\sum_{n=1}^{\infty} f_{n} e_{n}$, where $f_{n}: G \rightarrow \mathcal{C}$ are scalar-valued analytic functions, we get

$$
\left(\lambda_{n}-z\right) f_{n}(z)+<f(z), v>\alpha_{n}=0, \quad z \in G, \quad n \in \mathbb{N} .
$$

If we take $z=\lambda_{n} \in G \cap \Lambda(D)$ in the above equation, we obtain that $\left\langle f\left(\lambda_{n}\right), v>=0\right.$ for all $\lambda_{n} \in G \cap \Lambda(D)$. Since the set $\Lambda(D)$ is dense in $\overline{\Lambda(D)}$ and $G \subset \overline{\Lambda(D)}$, the set $G \cap \Lambda(D)$ is clearly dense in $G$. Hence $\langle f(z), v\rangle=0$ for all $z \in G$. Thus (14) implies that for every integer $n \in \mathbb{N}, f_{n}(z)=0$ for all $z \in G \backslash \Lambda(D)$. Since each $f_{n}$ is a continuous function and $G \backslash \Lambda(D)$ is dense in $G$, it follows that $f_{n}$ is identically equal to zero on $G$ for every $n \in \mathbb{N}$ and so is $f$.

\section{Contractions in $\mathcal{D}_{0}(\mathbb{D})$}

In this section we consider the class $\mathcal{D}_{0}(\mathbb{D})$ of the operators $T=D+u \otimes v \in \mathcal{D}_{0}$ for which $\Lambda(D) \subset \overline{\mathbb{D}}$. In this section we will characterize the contraction operators in $\mathcal{D}_{0}(\mathbb{D})$. The following proposition provides one such characterization and leads us to Corrolary 4.3 which gives a simple sufficient condition for an operator $T \in \mathcal{D}_{0}(\mathbb{D}) \cap \mathcal{D}_{2}$ to be a contraction.

Proposition 4.1. $T=D+u \otimes v \in \mathcal{D}_{0}(\mathbb{D})$ is a contraction operator if and only if

$$
\frac{|1-s<\widetilde{u}(s), D \widetilde{v}(s)>|}{\|\widetilde{u}(s)\|\|\widetilde{v}(s)\|}>\sqrt{s}, \quad s \in(0,1)
$$


where $\widetilde{u}(s)=\left(I-s D^{*} D\right)^{-1 / 2} u$ and $\widetilde{v}(s)=\left(I-s D^{*} D\right)^{-1 / 2} v$, or equivalently, in case $T \in$ $\mathcal{D}_{0}(\mathbb{D}) \cap \mathcal{D}_{2}$, if and only if

$$
\left|1-s \sum_{k=1}^{\infty} \frac{\alpha_{k} \overline{\beta_{k} \lambda_{k}}}{\left(1-s\left|\lambda_{k}\right|^{2}\right)}\right|^{2}>s\left(\sum_{k=1}^{\infty} \frac{\left|\alpha_{k}\right|^{2}}{\left(1-s\left|\lambda_{k}\right|^{2}\right)}\right)\left(\sum_{k=1}^{\infty} \frac{\left|\beta_{k}\right|^{2}}{\left(1-s\left|\lambda_{k}\right|^{2}\right)}\right), \quad s \in(0,1) .
$$

Proof. Clearly $T$ is a contraction if and only if $T^{*} T$ is a contraction. Since $T^{*} T$ is a positive selfadjoint operator, $T^{*} T$ is a contraction if and only if its spectrum is contained in the interval $[0,1]$. A simple computation shows that

$$
T^{*} T=D^{*} D+\left(D^{*} u+\|u\|^{2} v\right) \otimes v+v \otimes D^{*} u .
$$

Hence, $\sigma_{e}\left(T^{*} T\right)=\sigma_{e}\left(D^{*} D\right) \subset \sigma\left(D^{*} D\right) \subset[0,1]$ and so $T^{*} T\left(\sigma\left(T^{*} T\right)=\sigma_{e}\left(T^{*} T\right) \cup \sigma_{p}\left(T^{*} T\right)\right)$ has its spectrum contained in the interval $[0,1]$ if and only if its point spectrum does not intersect the interval $(1, \infty)$. We need the following lemma.

Lemma 4.2. Let $A \in \mathcal{L}(\mathcal{H})$ be invertible and $S=A+(a \otimes b)+(c \otimes d)$ for some vectors $a, b, c, d \in \mathcal{H}$. Then the following are equivalent:

(i) $S$ is not invertible,

(ii) $\operatorname{ker}(S) \neq \emptyset$,

(iii) the determinant of the matrix $\left[\begin{array}{cc}1+<A^{-1} a, b> & <A^{-1} c, b> \\ <A^{-1} a, d> & 1+<A^{-1} c, d>\end{array}\right]$ is zero.

Proof of Lemma 2.7. Since $S=A\left(I+\left(A^{-1} a \otimes b\right)+\left(A^{-1} c \otimes d\right)\right), S$ is not invertible if and only if $I+\left(A^{-1} a \otimes b\right)+\left(A^{-1} c \otimes d\right)$ is not invertible. Using the Fredholm theory, this latter operator being Fredholm of index zero, it is not invertible if and only if its kernel is not the (0) subspace. Hence (i) and (ii) are equivalent. For the equivalence of (ii) with (iii), let $x \in \mathcal{H}$ be a vector such that $S x=0$. This implies that $x+<x, b>A^{-1} a+<x, d>A^{-1} c=0$. Taking the inner product of this equation with $b$ and $d$ respectively, we get the following system of equations with the the unknowns $\langle x, b>$ and $\langle x, d\rangle$ :

$$
\left\{\begin{array}{l}
\left(1+<A^{-1} a, b>\right)<x, b>+<A^{-1} c, b><x, d>=0 \\
<A^{-1} a, d><x, b>+\left(1+<A^{-1} c, d>\right)<x, d>=0
\end{array}\right.
$$

Therefore if we assume that (ii) is true, then

$$
x=-<x, b>A^{-1} a-<x, d>A^{-1} c \neq 0
$$

and so at least one of the numbers $\langle x, b\rangle$ or $\langle x, d\rangle$ is not zero. This implies that the above homogeneous system has a nontrivial solution. This fact is equivalent with the statement (iii). Let us assume that (iii) is true. Then there is a nontrivial solution of the above homogeneous system of equations - say $\langle x, b\rangle=\alpha$ and $\langle x, d\rangle=\beta$. Hence $x=-\alpha A^{-1} a-\beta A^{-1} c$ is not the zero vector and a simple calculation shows that $\left(I+\left(A^{-1} a \otimes b\right)+\left(A^{-1} c \otimes d\right)\right) x=0$ or $S x=0$. 
We apply Lemma 2.7 for the case $A=D^{*} D-t I, a=D^{*} u+\|u\|^{2} v, b=c=v$, and $d=D^{*} u$, where $t \in \mathbb{R}, t>1$. Hence, $T^{*} T$ is a contraction if and only if the determinant of the matrix

$$
\left[\begin{array}{cc}
1+<\left(D^{*} D-t I\right)^{-1}\left(D^{*} u+\|u\|^{2} v\right), v> & <\left(D^{*} D-t I\right)^{-1} v, v> \\
<\left(D^{*} D-t I\right)^{-1}\left(D^{*} u+\|u\|^{2} v\right), D^{*} u> & 1+<\left(D^{*} D-t I\right)^{-1} v, D^{*} u>
\end{array}\right]
$$

equals zero for no $t \in(1, \infty)$. If we multiply the second column of this matrix by $\|u\|^{2}$ and subtract it from the first column, the determinant is the same as the determinant of the resulting matrix

$$
\left[\begin{array}{cc}
1+<\left(D^{*} D-t I\right)^{-1} D^{*} u, v> & <\left(D^{*} D-t I\right)^{-1} v, v> \\
<\left(D^{*} D-t I\right)^{-1} D^{*} u, D^{*} u>-\|u\|^{2} & 1+<\left(D^{*} D-t I\right)^{-1} v, D^{*} u>
\end{array}\right] .
$$

The $(2,1)$ entry can be written differently as follows:

$$
\begin{aligned}
& <\left(D^{*} D-t I\right)^{-1} D^{*} u, D^{*} u>-\|u\|^{2}=<\left(D^{*} D-t I\right)^{-1} D^{*} D u, u>-\|u\|^{2}= \\
& <\left(D^{*} D-t I\right)^{-1}\left(D^{*} D-t I\right) u, u>-\|u\|^{2}+t<\left(D^{*} D-t I\right)^{-1} u, u> \\
& =t<\left(D^{*} D-t I\right)^{-1} u, u>.
\end{aligned}
$$

If we observe that the $(1,1)$ entry is the complex conjugate of the $(2,2)$ entry, we obtain that $T^{*} T$ is a contraction operator if and only if the equation (in $t$ )

$$
\left|1+<\left(D^{*} D-t I\right)^{-1} D^{*} u, v>\right|^{2}-t<\left(D^{*} D-t I\right)^{-1} u, u><\left(D^{*} D-t I\right)^{-1} v, v>=0
$$

has no solution in the interval $(1, \infty)$. Finally, if we change variables by setting $s=1 / t$, $s \in(0,1)$, the above equation becomes

$$
\frac{\left|1-s<\left(I-s D^{*} D\right)^{-1} D^{*} u, v>\right|^{2}}{<\left(I-s D^{*} D\right)^{-1} u, u><\left(I-s D^{*} D\right)^{-1} v, v>}=s,
$$

which implies (15) since both members of the above equality are continuous functions of $s$ and the sign of the inequality is determined when $s=0$. The inequality (16) follows form (15) taking into account the explicit form of the operator $D$.

Corollary 4.3. Assume that for $T=D+(u \otimes v) \in \mathcal{D}_{0}(\mathbb{D}) \cap \mathcal{D}_{2}$ the coordinates of $u$ and $v$ satisfy the inequality

$$
\left(\sum_{k=1}^{\infty} \frac{\left|\alpha_{k}\right|^{2}}{\left(1-\left|\lambda_{k}\right|^{2}\right)}\right)\left(\sum_{k=1}^{\infty} \frac{\left|\beta_{k}\right|^{2}}{\left(1-\left|\lambda_{k}\right|^{2}\right)}\right) \leq 3-2 \sqrt{2} \simeq 0.171572876 \ldots
$$

Then $T$ is a contraction operator.

Proof. Using Proposition 4.1 we get that $T$ is a contraction operator if and only if

$$
\begin{gathered}
s\|\widetilde{u}(s)\|^{2}\|\widetilde{v}(s)\|^{2}< \\
1-2 s R e<\widetilde{u}(s), D \widetilde{v}(s)>+s^{2}|<\widetilde{u}(s), D \widetilde{v}(s)>|^{2},
\end{gathered}
$$

for every $s \in(0,1)$. We observe that (18) is satisfied if $\|\widetilde{u}(1)\|$ and $\|\widetilde{v}(1)\|$ are finite numbers satisfying

$$
\|\widetilde{u}(1)\|^{2}\|\widetilde{v}(1)\|^{2}+2\|\widetilde{u}(1)\|\|\widetilde{v}(1)\| \leq 1
$$


This last inequality is clearly satisfied if we have (17).

Corollary 4.4. Assume that $T=D+(u \otimes v) \in \mathcal{D}_{0}(\mathbb{D}) \cap \mathcal{D}_{2}$ is a contraction operator. Then the following inequality holds for every $s \in(0,1)$ :

$$
\left(\sum_{k=1}^{\infty} \frac{\left|\alpha_{k}\right|^{2}}{\left(1-s\left|\lambda_{k}\right|^{2}\right)}\right)\left(\sum_{k=1}^{\infty} \frac{\left|\beta_{k}\right|^{2}}{\left(1-s\left|\lambda_{k}\right|^{2}\right)}\right)<\frac{1}{s(1-\sqrt{s})^{2}}
$$

Proof. If $T$ is a contraction operator then we have (18), which implies that

$$
s\|\widetilde{u}(s)\|^{2}\|\widetilde{v}(s)\|^{2}<1+2\|\widetilde{u}(s)\|\|\widetilde{v}(s)\|+s^{2}\|\widetilde{u}(s)\|^{2}\|\widetilde{v}(s)\|^{2}, \quad s \in(0,1) .
$$

This last inequality is equivalent to (19) by simple computations.

\section{INVARIANT SUBSPACES}

If $A \in \mathcal{L}(\mathcal{H})$ and $x \in \mathcal{H}$ we write $\mathcal{C}_{x}(A)=\bigvee_{n=0}^{\infty}\left\{A^{n} x\right\}$. A vector $x \in \mathcal{H}$ is called cyclic for $A$ if $\mathcal{C}_{x}(A)=\mathcal{H}$. The following proposition characterizes those operators $T=D+(u \otimes v) \in \mathcal{D}_{0}$ for which $\operatorname{Lat}(T) \cap \operatorname{Lat}(D) \neq(0)$.

Proposition 5.1. If $T=D+(u \otimes v) \in \mathcal{D}_{0}$ then $D$ and $u \otimes v$ have a common n.i.s if and only if $\mathcal{C}_{u}(D) \neq \mathcal{H}$ or $\mathcal{C}_{v}\left(D^{*}\right) \neq \mathcal{H}$.

Proof. One can easily find all the invariant subspaces of $u \otimes v$. Namely, a subspace $\mathcal{S}$ is invariant for $u \otimes v$ if and only if $u \in \mathcal{S}$ or $v \perp \mathcal{S}$. Let us assume that $\mathcal{S}$ is a common n.i.s. for $D$ and $u \otimes v$. If $u \in \mathcal{S}$ we get that $\mathcal{C}_{u}(D) \neq \mathcal{H}$, and if $v \perp \mathcal{S}, \mathcal{S}^{\perp}$ is nontrivial invariant for $D^{*}$ containing $v$. Hence in this case $\mathcal{C}_{v}\left(D^{*}\right) \neq \mathcal{H}$. This proves the necessity. For the sufficiency, we just have to observe that $\mathcal{C}_{u}(D)$ and $\left(\mathcal{C}_{v}\left(D^{*}\right)\right)^{\perp}$ are common invariant subspaces for $D$ and $u \otimes v$.

The following proposition is a particular case of Bram's result [6] and answers the natural question whether an arbitrary diagonal operator admits a cyclic vector. For completeness we include here a simple proof of this fact which is a simplified version of the proof of Bram's result given in [6].

Proposition 5.2. Let $D=\operatorname{Diag}\left(\left\{\lambda_{n}\right\}\right) \in \mathcal{L}(\mathcal{H})$ such that every value in $\Lambda(D)$ has multiplicity one. Then there exits a cyclic vector for $D$.

Proof. We consider the operator $M_{z}$, the multiplication with the variable on $L^{2}(X, \eta)$, where $X=\overline{\Lambda(D)}$ and $\eta=\sum_{n=1}^{\infty} \frac{1}{n^{2}} \delta_{\lambda_{n}}$. Define $V: \mathcal{H} \rightarrow L^{2}(X, \eta)$ by $V x=f_{x}$ where $f_{x}(z)=n x_{n}$ if $z=\lambda_{n}$ and zero otherwise, $x=x_{1} e_{1}+x_{2} e_{2}+\ldots \in \mathcal{H}$. We have for each $x \in \mathcal{H},\|V x\|^{2}=\left\|f_{x}\right\|^{2}=\int_{X}\left|f_{x}(z)\right|^{2} d \eta(z)=\sum_{n=1}^{\infty} \frac{1}{n^{2}}\left|f_{x}\left(\lambda_{n}\right)\right|^{2}=\sum_{n=1}^{\infty}\left|x_{n}\right|^{2}=\|x\|^{2}$. Clearly, $V$ is an unitary operator and $V D V^{-1}=M_{z}$, which implies that it suffices to show that $M_{z}$ has a cyclic vector. For each $n \in \mathbb{N}$, denote $K_{n}=\left\{\lambda_{1}, \lambda_{2}, \ldots, \lambda_{n}\right\}$. Since all the eigenvalues $\lambda_{n}$ are assumed to be distinct, the following system of linear equations has a unique solution in $c_{0}, c_{1}, \ldots, c_{n}$ :

$$
\overline{\lambda_{j}}=c_{0}+c_{1} \lambda_{j}+\ldots+c_{n} \lambda_{j}^{n}, \quad j=1,2, \ldots, n .
$$


Let $p_{n}(z)=c_{0}+c_{1} z+\ldots+c_{n} z^{n}$, where the coefficients $c_{0}, c_{1}, \ldots, c_{n}$ are satisfying (20). Using this notation, (20) can by written as $\bar{z}=p_{n}(z)$ on $K_{n}$. We now construct a Borel measure $\nu$ on $X$ with the following properties:

(a) $\nu$ is a measure absolutely continuous with respect to $\eta$,

(b) $\frac{d \nu}{d \eta}:=\phi$ is essentially bounded $([\eta])$,

(c) the function $1(z)=1$ is a cyclic vector for $M_{z}$ acting on $L^{2}(X, \nu)$.

First we choose $\left.a_{n}=\left(\max _{1 \leq k \leq n}\left[\sup _{z \in X}\left|p_{k}(z)\right|^{2}\right)\right]\right)^{-1}$ for each $n \in \mathbb{N}$, and let then $\nu=\delta_{\lambda_{1}}+\sum_{n=2}^{\infty} \frac{1}{n^{2}} a_{n-1} \delta_{\lambda_{n}}$. Clearly, $a_{1} \geq a_{2} \geq \ldots \geq a_{n}>0$. It is easy to observe that (a) is satisfied, and in order to check the second property we take $\phi(z)=a_{n-1}$ if $z=\lambda_{n}, n \geq 2$, 1 if $z=\lambda_{1}$ and zero anywhere else. Hence, $0 \leq \phi(z) \leq \max \left\{a_{1}, 1\right\}=a_{0}$ for every $z \in X$. To check the third property, we want to show that $p_{n}$ converges in $L^{2}(X, \nu)$ to the function $z \rightarrow \bar{z}:$

$$
\begin{gathered}
\int_{X}\left|\bar{z}-p_{n}(z)\right|^{2} d \nu(z)=\int_{X \backslash K_{n}}\left|\bar{z}-p_{n}(z)\right|^{2} d \nu(z) \leq 2 \int_{X \backslash K_{n}}|\bar{z}|^{2} d \nu(z)+ \\
2 \int_{X \backslash K_{n}}\left|p_{n}(z)\right|^{2} d \nu(z) \leq 2 \int_{X \backslash K_{n}} \phi d \eta+2\left\|p_{n}\right\|_{\infty}^{2} \int_{X \backslash K_{n}} \phi d \eta \leq \\
2 a_{0} \eta\left(X \backslash K_{n}\right)+2\left\|p_{n}\right\|_{\infty}^{2} a_{n} \eta\left(X \backslash K_{n}\right) \leq 2\left(a_{0}+1\right) \eta\left(X \backslash K_{n}\right) \rightarrow 0,
\end{gathered}
$$

as $n \rightarrow \infty$. In other words, this means that the sequence of functions $z \rightarrow p_{n}\left(M_{z}\right) 1(z)$ converges in $L^{2}(X, \nu)$ to the function $z \rightarrow \bar{z}$. From here, we obtain that for any polynomial $q \in \mathbb{C}[z]$, the sequence $\left(q p_{n}\right)\left(M_{z}\right) 1(\cdot)$ converges to $z \rightarrow q(z) \bar{z}$. Thus, the function $z \rightarrow(\bar{z})^{2}$ is in $\mathcal{C}_{1(\cdot)}\left(M_{z}\right)$. Inductively, we can show that $z \rightarrow(\bar{z})^{n} \in \mathcal{C}_{1(\cdot)}\left(M_{z}\right)$ for every $n \in \mathbb{N}$. Finally, $p(z, \bar{z}) \in \mathcal{C}_{1(\cdot)}\left(M_{z}\right)$ for every polynomial in two variables $p(z, \bar{z})$, and by Stone-Weierstrass theorem we get that any continuous function on $X$ is in $\mathcal{C}_{1(\cdot)}\left(M_{z}\right)$. This shows that the property (c) holds.

Now, we want to show that $\phi^{1 / 2}$ is a cyclic vector for $M_{z}$ acting on $L^{2}(X, \eta)$. If $f \in L^{2}(X, \eta)$ then clearly $\frac{f}{\phi^{1 / 2}}$ is in $L^{2}(X, \nu)$ and hence it can be approximated by a sequence of polynomials $q_{n}$ in $L^{2}(X, \nu)$. Therefore,

$$
\begin{gathered}
\int_{X}\left|q_{n}(z) \phi^{1 / 2}-f(z)\right|^{2} d \eta(z)=\int_{X} \phi(z)\left|q_{n}(z)-\frac{f(z)}{\phi^{1 / 2}(z)}\right|^{2} d \eta(z)= \\
\int_{X}\left|q_{n}(z)-\frac{f(z)}{\phi^{1 / 2}(z)}\right|^{2} d \nu(z) \rightarrow 0,
\end{gathered}
$$

by our assumption. This proves that $q_{n}\left(M_{z}\right) \phi^{1 / 2}$ converges to $f$ in $L^{2}(X, \eta)$ which finishes the proof.

Let us observe that if $T=D+(u \otimes v) \in \mathcal{D}_{2}$ we have $\pi(T)=\pi(D)$, and hence, since $\pi(D)$ is normal in the Calkin algebra, we have that $\sigma_{l e}(D)=\sigma_{r e}(D)$ and consequently $\sigma_{l e}(T)=\sigma_{r e}(T)=\sigma_{e}(T)=\sigma_{e}(D)=\Lambda(D)^{\prime}$. Hence, well-known reductions of the invariant subspace problem (see [5] for part (iii)) applied to our particular case and together with what we have proved so far give the following proposition.

Proposition 5.3. If $T=D+(u \otimes v) \in \mathcal{D}_{2}$, and 
(i) $\sigma(T) \neq \Lambda(D)^{\prime}$ (equivalently $1 \in f_{T}^{-1}(\mathbb{C} \backslash \bar{\Lambda})$ ), or

(ii) $\Lambda(D)^{\prime}$ is not connected, or

(iii) $\Lambda(D)^{\prime}$ is a singleton, or

(iv) $u[$ resp. $v]$ is not cyclic for $D\left[\right.$ resp. $\left.D^{*}\right]$,

then $T$ has a n.h.s.

When one searches for invariant subspaces for an operator $T$ it is useful to have a description of its commutant $\{T\}^{\prime}:=\{A \in \mathcal{L}(\mathcal{H}): A T=T A\}$.

Proposition 5.4. Let $T=D+(u \otimes v) \in \mathcal{D}_{2}$, and $A \in \mathcal{L}(\mathcal{H})$. Then $A \in\{T\}^{\prime}$ if and only if there exist a sequence of complex numbers $\left\{t_{n}\right\}_{n \in \mathbb{N}}$ and a positive constant $C$ such that

(i) for every square-summable sequence $\left\{\xi_{k}\right\}_{k \geq 1}$ we have

$$
\sum_{n}\left|\alpha_{n}\right|^{2}\left|\sum_{k \geq 1, k \neq n}^{\infty} \xi_{k} \bar{\beta}_{k} \gamma_{k, n}\right|^{2} \leq C \sum_{n}\left|\xi_{n}\right|^{2},
$$

where $\gamma_{k, n}:=\frac{t_{k}-t_{n}}{\lambda_{k}-\lambda_{n}}$, for $k \neq n,(k, n \in \mathbb{N})$,

(ii) for every $k \in \mathbb{N}$,

$$
A e_{k}=s_{k} e_{k}+\bar{\beta}_{k} \sum_{n \geq 1, n \neq k} \alpha_{n} \gamma_{k, n} e_{n}
$$

where the sequence defined by

$$
s_{k}=t_{k}-\sum_{n \geq 1, n \neq k} \alpha_{n} \overline{\beta_{n}} \gamma_{k, n}, \quad k \in \mathbb{N}
$$

is a bounded sequence.

Proof. The equality $A T=T A$ can be written equivalently as

$$
A D-D A=\left(u \otimes A^{*} v\right)-(A u \otimes v) .
$$

For the necessity part, let $\left\{t_{k}\right\}$ be defined by the equation $A u=\sum_{k=1}^{\infty} t_{k} \alpha_{k} e_{k}$. For every integer $k \geq 1$, we have $<(A D-D A) e_{k}, e_{k}>=0$ and then from (24) we obtain

$$
<e_{k}, A^{*} v><u, e_{k}>-<e_{k}, v><A u, e_{k}>=0,
$$

which in turn implies that $<e_{k}, A^{*} v>=t_{k} \bar{\beta}_{k}$. Hence, using (24) again, we get

$$
\left(\lambda_{k}-D\right) A e_{k}=\bar{\beta}_{k}\left(t_{k} u-A u\right)=\bar{\beta}_{k} \sum_{n \geq 1, n \neq k}^{\infty} \alpha_{n}\left(t_{n}-t_{k}\right) e_{n}, \quad k \geq 1
$$

which implies that we can express $A e_{k}$ as in (22). Taking the inner product of both sides of (22) with $v$, we obtain that $s_{k}$ is given by (23). To obtain the inequality (21) we first need to observe that $s_{k}=<A e_{k}, e_{k}>$ (by (22) and so $\left\{s_{k}\right\}$ is a bounded sequence. Thus, the inequality (21) follows easily from the boundedness of the operator $A-\widetilde{D}$, where $\widetilde{D}$ is the diagonal operator defined by $\widetilde{D} e_{k}=s_{k} e_{k}, k \in \mathbb{N}$. 
For sufficiency, we observe that the linear operator $A$ defined by (22) is bounded because of (21) and the hypothesis that $\left\{s_{k}\right\}$ is bounded. Then from (22) and (23) we get that $<e_{k}, A^{*} v>=t_{k} \bar{\beta}_{k}$ and $A u=\sum_{k=1}^{\infty} t_{k} \alpha_{k} e_{k}$. Using these two relations and (22), we obtain (25) which is equivalent to (24).

Next we would like to combine Proposition 5.4 with Lomonosov's theorem (cf. [21]) to obtain sufficient conditions for existence of n.i.s. for operators in $\mathcal{D}_{2}$. For this purpose we introduce some more notation. Let $\mathcal{H}(U)$ be the set of analytic functions on the open set $U(\subset \mathbb{C})$. For a fixed $w \in U$ we define a linear transformations on $\mathcal{H}(U)$, $\psi \rightarrow \Gamma(\psi)(\cdot, w)$, by

$$
\Gamma(\psi)(z, w)=\left\{\begin{array}{l}
\frac{\psi(z)-\psi(w)}{z-w} \text { if } z \neq w \\
\phi^{\prime}(w) \text { if } z=w,
\end{array} \quad z \in U, \quad \psi \in \mathcal{H}(U) .\right.
$$

For $T \in \mathcal{D}_{2}$ given by (1), and $U$ such that $\overline{\Lambda(D)} \subset U$ we define another linear transformation on $\mathcal{H}(U)$ by

$$
B_{T}(\psi)(z)=\int_{\Lambda(D)} \Gamma(\psi)(z, w) d \nu(w), \quad z \in U \cup \Lambda(D), \quad \psi \in \mathcal{H}(U),
$$

where $\nu$ is the atomic measure supported on $\Lambda(D)$ given by $\nu=\sum_{n \geq 1} \alpha_{n} \overline{\beta_{n}} \delta_{\lambda_{n}}$.

Theorem 5.5. Let $T \in \mathcal{D}_{2}$ given by (1) and $B_{T}$ defined by (27). Suppose there exists a function $\psi \in \mathcal{H}(U)$, with $U \supset \overline{\Lambda(D)}$, such that $B_{T} \psi=\psi$ and $\psi$ is not zero on $\overline{\Lambda(D)}$. Then $T$ has a nontrivial invariant subspace.

Proof. Let us consider $t_{n}=\psi\left(\lambda_{n}\right), n \in \mathbb{N}$, and let $A_{\psi}$ be the operator $A$ defined as in (22) and (23). We will show that $A_{\psi}$ satisfies (21) and it is a nonzero compact operator. By Proposition 5.4, $T$ commutes with a nonzero compact operator and then using Lomonosov's theorem $T$ admits a n.i.s.

Suppose that $A_{\psi}=0$. Then, from the proof of Proposition 5.4, we have $A_{\psi} u=$ $\sum_{n \in \mathbb{N}} \alpha_{n} t_{n} e_{n}$, and so $t_{n}=0$ for all $n \in \mathbb{N}$. By Proposition 5.3 we can assume that $\sigma(T)=$ $\Lambda(D)^{\prime}$ and $\Lambda(D)^{\prime}$ is connected. Thus we can consider $\widetilde{U}$ to be the connected component of $U$ containing $\Lambda(D)^{\prime}$. Hence, $\psi=0$ on $\widetilde{U}$ since $\Lambda(D)$ must have an accumulation point in $\Lambda(D)^{\prime} \subset \widetilde{U}(\widetilde{U}$ is connected $) . \mathbb{C} \backslash \widetilde{U}$ cannot contain but finitely many points of $\Lambda(D)$ where $\psi$ must be zero because $t_{n}=0, n \in \mathbb{N}$. This contradicts our assumption on $\psi$, and so $A_{\psi}$ is not zero.

Since $\psi \in \mathcal{H}(U)$ and $\overline{\Lambda(D)} \subset U$, there exists a constant $C_{1}>0$ such that $|\Gamma(\psi)(z, w)| \leq C_{1}$ for all $z, w \in \overline{\Lambda(D)}$ and so, with the notation from Proposition 5.4, $\left|\gamma_{k, n}\right| \leq C_{1}$ for every $k, n \in \mathbb{N}, k \neq n$. Then, using Cauchy's inequality, we have

$$
\sum_{n}\left|\alpha_{n}\right|^{2}\left|\sum_{k \geq 1, k \neq n} \xi_{k} \bar{\beta}_{k} \gamma_{k, n}\right|^{2} \leq C_{1}^{2} \sum_{n}\left|\alpha_{n}\right|^{2} \sum_{k \geq 1, k \neq n}\left|\xi_{k}\right|^{2} \sum_{k \geq 1, k \neq n}\left|\beta_{k}\right|^{2} \leq C \sum_{k}\left|\xi_{k}\right|^{2},
$$


where $C=C_{1}^{2}\|u\|^{2}\|v\|^{2}$. This proves that inequality (21) is satisfied. Also, the sequence defined by (23) is bounded since $\left\{t_{n}\right\}$ is clearly bounded and for every $k \in \mathbb{N}$

$$
\left|\sum_{n \geq 1, n \neq k} \alpha_{n} \bar{\beta}_{n} \gamma_{k, n}\right| \leq C_{1}\|u\|\|v\| .
$$

Then, by Proposition 5.4, $A_{\psi}$ commutes with $T$. From (23), for every $k \in \mathbb{N}$ we have

$$
s_{k}=\psi\left(\lambda_{k}\right)-\sum_{n \geq 1, n \neq k} \alpha_{n} \overline{\beta_{n}} \gamma_{k, n}=\psi\left(\lambda_{k}\right)-B_{T}(\psi)\left(\lambda_{k}\right)+\alpha_{k} \overline{\beta_{k}} \psi^{\prime}\left(\lambda_{k}\right),
$$

which simplifies to $s_{k}=\alpha_{k} \overline{\beta_{k}} \psi^{\prime}\left(\lambda_{k}\right)$ because of our hypothesis on $\psi$. Clearly, $\lim _{k \rightarrow \infty} s_{k}=0$ and so the diagonal operator $\widetilde{D}\left(\widetilde{D} e_{k}=s_{k} e_{k}, k \in \mathbb{N}\right)$ is a compact operator. Since $A_{\psi}=$ $\widetilde{D}+B$ where $B$ is defined by

$$
B e_{k}=\bar{\beta}_{k} \sum_{n \geq 1, n \neq k} \alpha_{n} \gamma_{k, n} e_{n}, \quad k \in \mathbb{N},
$$

it suffices to show that $B$ is a compact operator. In fact, $B$ is a Hilbert-Schmit operator since

$$
\sum_{k \in \mathbb{N}}\left\|B e_{k}\right\|^{2}=\sum_{k \in \mathbb{N}}\left|\beta_{k}\right|^{2} \sum_{n \geq 1, n \neq k}\left|\alpha_{k}\right|^{2}\left|\gamma_{k, n}\right|^{2}<C,
$$

which finishes our proof.

Corollary 5.6. Let $T \in \mathcal{D}_{2}$ given by (1) such that $\Lambda(D) \subset \overline{\mathbb{D}}$. Suppose that $f_{T}(c f .(3))$ is bounded on $\mathbb{C} \backslash \mathbb{D}$ and let $T_{\phi}$ be the Toeplitz operator on $H^{2}(\mathbb{D})$ of symbol $\phi(\zeta)=\overline{f_{T}(\bar{\zeta})}$ for $\zeta \in \partial \mathbb{D}$. In addition we assume that the equation $T_{\phi}(\psi)=\psi$ has a solution $\psi \in H^{2}(\mathbb{D})$ which is analytic on an open set $U \supset \overline{\mathrm{D})}$ and not zero on $\overline{\Lambda(D)}$. Then there exists a n.i.s for $T$.

Proof. The assumption on $f_{T}$ insures that $\phi$ is in $L^{\infty}(\partial \mathrm{DD})$ and so the Toeplitz operator $T_{\phi}$ is well defined. Indeed, for $z \in \mathbb{D}$ we have $f_{T}\left(\frac{1}{z}\right)=z \sum_{n=1}^{\infty} \frac{\alpha_{n} \overline{\beta_{n}}}{1-z \lambda_{n}}=\sum_{k=0}^{\infty} m_{k} z^{k+1}$, where $m_{k}$ are the moments of the measure $\nu$ ( i.e., $m_{k}=\int_{\mathbb{D}} \zeta^{k} d \nu(\zeta), k \in \mathbb{N} \cup\{0\}$ ). So, $z \rightarrow f_{T}(1 / z)$ is a bounded analytic function on $\mathbb{D}$, and thus $\phi \in L^{\infty}(\partial \mathbb{D})$. In fact, $T_{\phi}$ is a co-analytic Toeplitz operator. We want to show that $B_{T}$ and $T_{\phi}$ act the same way on functions $\psi \in H^{2}(\mathbb{D})$ which are analytic on open neighborhoods of $\overline{\mathbb{D}}$. Forsooth, if $\psi(z)=\sum_{k=0}^{\infty} a_{k} z^{k} \in H^{2}(\mathbb{D})$ is such a function, we have

$$
\begin{gathered}
T_{\phi}(\psi)\left(e^{i \theta}\right)=P_{H^{2}}\left(\phi\left(e^{i \theta}\right) \psi\left(e^{i \theta}\right)\right)=P_{H^{2}}\left(\sum_{k=0}^{\infty} m_{k} e^{-i(k+1) \theta} \sum_{l=0}^{\infty} a_{l} e^{i l \theta}\right)= \\
P_{H^{2}}\left(\sum_{k, l \geq 0} m_{k} a_{l} e^{i(l-k-1) \theta}\right)=\sum_{n=0}^{\infty}\left(\sum_{k=0}^{\infty} m_{k} a_{n+k+1}\right) e^{i n \theta} \text { in } H^{2}(\mathbb{D}) .
\end{gathered}
$$

On the other hand, if $z \in \mathbb{D} \backslash \Lambda(D)$ we have 


$$
\begin{gathered}
B_{T}(\psi)(z)=\sum_{k=1}^{\infty} \frac{\psi(z)-\psi\left(\lambda_{k}\right)}{z-\lambda_{k}} \alpha_{k} \overline{\beta_{k}}=\sum_{k=1}^{\infty} \sum_{n=1}^{\infty} a_{n}\left(z^{n-1}+\ldots+\lambda_{k}^{n-1}\right) \alpha_{k} \overline{\beta_{k}}= \\
\sum_{n=1}^{\infty} a_{n}\left(z^{n-1}+m_{1} z^{n-2}+\ldots+m_{n-1}\right)=\sum_{k=0}^{\infty}\left(\sum_{n=0}^{\infty} m_{n} a_{n+k_{+}}\right) z^{k} .
\end{gathered}
$$

The assumptions on $\psi$ allows one to do the computations in (28) and (29). Moreover, if $T_{\phi}(\psi)=\psi$, comparing (28) with $(29)$ we have $B_{T}(\psi)(z)=\psi(z)$ for $z \in \mathbb{D}$, and so we can apply Theorem 5.5 to conclude the corollary.

\section{REFERENCES}

[1] N. Benamara and N. Nikolski, Resolvent tests for similarity to normal operators, Proc. London Math. Soc. 78(1999), 585-626.

[2] L. Brown, A. Shields and K. Zeller, On absolutely convergent exponential sums, Trans. Amer. Math. Soc., 96(1960), 162-183.

[3] K. Clancey, Seminormal operators, Lecture Notes in Math., vol. 742, Springer-Verlag, New York, 1979.

[4] D. Clark, One-dimensional perturbations of restricted shifts, J. Analyse Math. 25(1972), $169-191$.

[5] I. Colojoara and C. Foias, Theory of generalized spectral operators, Science Publishers, New York, 1968.

[6] J. B. Conway, A course in functional analysis, Springer-Verlag, New York, 1985.

[7] C. Davis, Eigenvalues of compressions, Bull. Math. de la Soc. Sci. Math. Phys. de la R.P.Roumaine (N.S.), 3(51) (1959), 3-5.

[8] R. Del Rio, S. Jitomirskaya, Y. Lasta, and B. Simon, Operators with singular continuous spectrum. IV. Hausdorff dimensions, rank one perturbations, and localization, J. Anal. Math. 69(1996), 153-200.

[9] R. Del Rio, N. Makarov and B. Simon, Operators with singular continuous spectrum. II. Rank one operators, Comm. Math. Phys. 165(1994), 59-67.

[10] R. Del Rio and B. Simon, Point spectrum and mixed spectral types for rank one perturbations, Proc. Amer. Math. Soc., 125(1997), 3593-3599.

[11] W. F. Donoghue, On the perturbation of spectra, Comm. Pure. Appl. Math. 18(1965), 559-579.

[12] J. Eschmeier and M. Putinar, Bishop's condition $(\beta)$ and rich extensions of linear operators, Indiana Univ. Math. J., 37 (1988), 325-348.

[13] K. Fan and G. Pall, Imbedding conditions for Hermitian and normal matrices, Canad. J. Math. 9(1957), 298-304.

[14] S. Hassi and H. de Snoo, On rank one perturbations of selfadjoint operators, Integral Equations Operator Theory, 29(1997), 288-300.

[15] S. Hassi, H. de Snoo, and A. Willemsma, Smooth rank one perturbations of selfadjoint operators, Proc. Amer. Math. Soc. 126(1998), 2663-2675.

[16] H. Hochstadt, One dimensional perturbations of compact operators, Proc. Amer. Math. Soc. 37(1973), 465-467.

[17] G. Islamov, Properties of one-rank perturbations, Izv. Vyssh. Uchebn. Zaved. Mat. 4(1989), 29-35.

[18] W. Johnston, A condition for absence of singular spectrum with an application to perturbations of selfadjoint Toeplitz operators, Amer. J. Math. 113 (1991), 243-267.

[19] V. Kapustin, One-dimensional perturbations of singular unitary operators, Zap. Nauchn. Sem. S.Peterburg. Otdel. Mat. Inst. Steklov. (POMI) 232 (1996), 118-122, 216.

[20] T. Kato, Perturbation theory for linear operators, Springer-Verlag, New York, 1976.

[21] V. Lomonosov, On invariant subspaces of families of operators commuting with a completely continuous operator, Funk. Anal. i Prilozen 7(1973), 55-56 (Russian).

[22] N. G. Makarov, One-dimensional perturbations of singular unitary operators, Acta Sci. Math. 42(1988), 459-463.

[23] N. G. Makarov, Perturbations of normal operators and the stability of the continuous spectrum, Izv. Akad. Nauk SSSR Ser. Mat. 50 (1986), 1178-1203, 1343. 
[24] M. Malamud, Remarks on the spectrum of one-dimensional perturbations of Volterra, Mat. Fiz. No. 32 (1982), 99-105.

[25] Y. Mikityuk, The singular spectrum of selfadjoint operators, (Russian) Dokl. Akad. Nauk SSSR 303 (1988), 33-36; translation in Soviet Math. Dokl. 38 (1989), 472-475.

[26] L. Mirsky, Matrices with prescribed characteristic roots and diagonal elements, J. London Math. Soc. 33(1958), 14-21.

[27] B. Sz.-Nagy and C. Foias, Harmonic analysis of operators on Hilbert space, American Elsevier, New York, 1970.

[28] Nakamura, Yoshihiro, One-dimensional perturbations of the shift, Integral Equations Operator Theory 3(1993), 337-403.

[29] B. Simon, Spectral analysis of rank one perturbations and applications. Mathematical quantum theory. II. Schrdinger operators, CRM Proc. Lecture Notes, 8 Amer. Math. Soc., Providence, RI, (1995), 109-149.

[30] B. Simon, Operators with singular continuous spectrum. VII. Examples with borderline time decay, Comm. Math. Phys. 176(1996), 713-722.

[31] J. G. Stampfli, One-dimensional perturbations of operators, Pacific J. Math., 115(1984), 481-491.

[32] H. Vasudeva, One dimensional perturbations of compact operators, Proc. Amer. Math. Soc. 57 (1976), 58-60.

[33] J. Wolff, Sur les séries $\sum A_{k} /\left(z-\alpha_{k}\right)$, C. R. Acad. Sci. Paris, 173 (1921), 1057-158, 1327-1328.

The University of Georgia

Department of Mathematics

Athens, GA 30602, USA

e-mail: ionascu@alpha.math.uga.edu

Mathematical Subject Classification: Primary 47Axx; Secondary 47A15, 47A55, $30 \mathrm{~B} 50$. 